\title{
A cross-cultural analysis of salivary cortisol patterns in breast cancer survivors
}

\author{
Cynthia Wan*,1, Kayla Boileau1', Danielle D'Amico², Vivian Huang², Alexandra J \\ Fiocco $^{2}$, Richard Clément ${ }^{1} \&$ Catherine Bielajew ${ }^{1}$ \\ ${ }^{1}$ School of Psychology, University of Ottawa, Ottawa, Canada \\ ${ }^{2}$ Department of Psychology, Ryerson University, Toronto, Canada \\ *Author for correspondence: cwan062@uottawa.ca
}

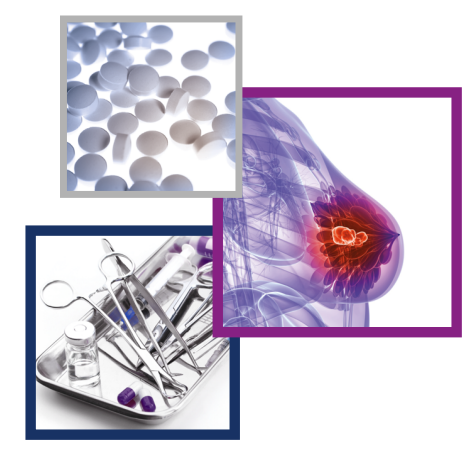

\begin{abstract}
Aim: In this study, we examined whether Chinese and White women with and without a history of breast cancer exhibit differences in physiological and psychological stress profiles. Methods: Diurnal and reactive salivary cortisol profiles and psychological stress patterns of 41 breast cancer survivors and 58 healthy women were assessed. Results: Breast cancer survivors displayed a blunted acute cortisol response but there was no main effect of ethnocultural membership. Subjective appraisals of stress during the acute stressor revealed a significant interaction between ethnocultural group, health status and time $(p=0.032)$. Conclusion: Our results support the existing literature though suggest group differences in the appraisal of stress; thus, underscoring the importance of cultural sensitivity and awareness among clinicians and existing programs.
\end{abstract}

First draft submitted: 23 January 2019; Accepted for publication: 21 March 2019; Published online: 16 April 2019

Keywords: acute cortisol $\bullet$ breast cancer survivorship $\bullet$ cultural orientation $\bullet$ diurnal cortisol $\bullet$ physiological stress $\bullet$ psychological stress $\bullet$ reactivity $\bullet$ salivary cortisol

Breast cancer is increasingly diagnosed in Asian-American women [1]. Researchers have suggested several factors that account for this, such as shifts in risk factor profiles [2], westernized lifestyles [3] and acculturative stress [4]. To better understand the breast cancer survivorship experience in Asian women, more and more studies are investigating the physiological and psychological effects of breast cancer in this group as a means of understanding ethnocultural differences in survivorship trajectory [5-9].

\section{Stress \& health}

Stress is a universal experience, the product of an interaction between an individual and the environment, namely the individual's appraisal of the environment and coping capabilities [10]. Thus, stress can be viewed as positive, negative or neutral and beneficial toward promoting one's survival [11]. In response to a stressor, the hypothalamicpituitary-adrenal (HPA) axis is activated to prepare the organism to deal with environmental challenges [12], which initiates the production of glucocorticoids such as cortisol. Cortisol is a hormone that regulates bodily functions, such as homeostasis and immune response [13], and is commonly measured via plasma, serum, urine or saliva [14].

Cortisol has a relatively stable diurnal pattern in healthy individuals $[15,16]$. It reaches its peak approximately 30 to $60 \mathrm{~min}$ after waking, thereafter steadily declining throughout the day [15]. Cortisol secretion and basal levels can be affected by numerous factors, such as hormonal status [17], age and sex [18], circadian rhythm [19] and illnesses such as cancer [20].

While there are many studies assessing the cortisol profile of women with breast cancer during their treatment [21,22], very few have focused on post-treatment biomarker patterns [6,23,24]. Based on the limited findings, most studies report no significant differences in diurnal cortisol patterns between breast cancer survivors and healthy women [23,24]. However, in metastatic breast cancer survivors, a flattened profile and/or differences in peaks and troughs in the diurnal rhythm $[25,26]$.

Future Medicine 
In contrast, cortisol patterns are markedly different in acute stress situations compared with that of daily patterns. In response to an acute stressor, cortisol secretion typically increases and returns to basal levels within $1-2 \mathrm{~h}$ after cessation [27]; however, breast cancer survivors exhibit a blunted cortisol response to an acute stressor, which has been interpreted as a consequence of chronic inflammation and chronic stress - the continuous and prolonged activation of the HPA axis — resulting in a hyporeactive HPA axis $[24,28]$.

\section{Acculturative stress \& cortisol rhythms}

Acculturative stress is defined as a stress response to various acculturation-related stressors (e.g., learning a new language, set of values, or behaviors) and life events $[4,29]$ and may be experienced by first-, second- and thirdgeneration immigrants [30,31].

Many studies acknowledge the impact that acculturative stress has on an individual's psychological and physiological health [32-34]. For instance, Choi noted that high levels of acculturative stress were associated with depressive symptomology among Korean Americans [32]. Furthermore, Logan et al. found that acculturative stress was related to arterial stiffness, demonstrating a marginally significant relationship between acculturative stress and diastolic blood pressure [34].

Based on existing stress literature, which predominantly examines the stress responses of White breast cancer survivors, it is often assumed that breast cancer in Chinese women also leads to a dysregulated HPA axis. Thus, the focus of studies is often on the efficacy of various interventions on the stress system and/or their qualitative survivorship experience. Recently, Ho et al. noted that Chinese breast cancer survivors exhibited a flatter diurnal cortisol slope [22]. Furthermore, they observed an association between the slope of the diurnal cortisol rhythm with higher negative social support and poorer perceived health. Despite these findings, to our knowledge, there are no empirical studies investigating the differences in HPA axis reactivity between Chinese and White women with and without a prior breast cancer diagnosis, whilst considering the effects of various psychosocial variables, such as cultural orientation.

To address the gaps in the literature, our study investigated whether ethnocultural membership and health status influences one's physiological and psychological stress patterns. To do so, we examined differences in diurnal and acute cortisol patterns, as well as psychological patterns of stress between Chinese and White women with and without a prior diagnosis of breast cancer. Following a similar study design to the one used by Couture-Lalande et al. [24], we expected to see a replication of results: breast cancer survivors, irrespective of ethnocultural group membership, would exhibit a blunted acute cortisol response and display diurnal patterns of cortisol reactivity comparable to that of healthy control participants. With the added complexity of acculturative stress, we further hypothesized that the dysregulation in acute stress response in Chinese breast cancer survivors would be more pronounced than that of White breast cancer survivors.

\section{Methods}

Participants

Chinese and White women with and without a prior diagnosis of breast cancer were recruited from Ottawa and Toronto (ON, Canada) via online classifieds (e.g., Kijiji, comefromchina.com) and through various community and cancer support groups (e.g., Babes4Breast, Breast Cancer Action Ottawa, Ottawa Regional Cancer Foundation). A total of 69 healthy women (31 Chinese, 38 White) and 50 breast cancer survivors (20 Chinese, 30 White) were recruited for the study. Due to missing data, our analyses included a final sample of 58 healthy women (27 Chinese, 31 White) and 41 breast cancer survivors (17 Chinese, 24 White). Sample sizes were based on prior power analyses of similar studies conducted in the laboratory [7,24], which revealed that a sample size of between 22 and 25 was sufficient to detect significant group differences in stress profiles.

Individuals were included in the study if they met the following eligibility criteria: A woman of Chinese or White descent; between the ages of 30 and 80; currently residing in either Ottawa or Toronto; and able to read and understand English. In addition to these criteria, to be eligible for the breast cancer survivor group, individuals must also have had a prior diagnosis of breast cancer; have completed all systemic treatment at least 6 months prior to the study start date (hormonal treatment excepted); and be currently considered cancer free. Individuals were excluded if they had a previous diagnosis of other cancers (noninvasive skin cancer and cervical cancer excepted); have or have had a debilitating psychiatric or medical illness (other than breast cancer); and were pregnant at the time of the study. Participants were compensated $\$ 50$ for their involvement in the study. 


\section{Measures}

\section{Sociodemographic questionnaire}

We developed the sociodemographic questionnaire for other studies in our laboratory [5-8,24]; it includes questions relating to age, marital status, education and income. We added questions regarding participants' place of birth, their age of immigration (if applicable) and number of years spent in Canada. The SDQ also has items pertaining to medical characteristics and habits known to influence cortisol concentrations (e.g., smoking, teeth brushing). Breast cancer survivors were asked to complete additional items related to their breast cancer history (e.g., age of diagnosis, stage, treatment).

\section{General Ethnicity Questionnaire - Abridged}

The General Ethnicity Questionnaire (GEQ) [35] is a 77-item questionnaire that measures cultural orientation, that is, the degree to which an individual identifies with a dominant and nondominant culture by assessing the extent to which they participate in the activities, traditions and lifestyle of the two cultures. Questions pertaining to orientation toward Canadian (GEQCA, dominant culture) and Chinese (GEQCH, non-dominant culture) cultures were identical in wording with the exception of the culture being referenced. The scale measures six domains of acculturation: language use and proficiency, social affiliation, cultural participation, cultural pride, cultural exposure and preference for cultural food and media. Participants rated the items on a 5-point Likert-type scale, ranging from 1 (strongly disagree) to 5 (strongly agree). Higher scores reflect higher orientation toward that particular culture. Tsai et al. [35] assessed cultural orientation toward Chinese (GEQCH) and American (GEQA) cultures, and they reported that the GEQ had high internal reliability ( $\alpha=0.92$ for both scales). Test-retest reliability was 0.62 $(\mathrm{SD}=0.22$; GEQCH$)$ and $0.57(\mathrm{SD}=0.16$; GEQA). In the present study, the GEQ demonstrated high internal reliability for all groups ( $\alpha=0.88$ to 0.93 , GEQCA; and 0.86 to 0.93 , GEQCH).

\section{Bidimensional Fatigue Scale}

The Bidimensional Fatigue Scale (BFS) [36] is a 14-item questionnaire that assesses the degree of physical and mental fatigue experienced within the past two weeks. Participants rate items such as 'do you feel sleepy or drowsy?' on a 4-point scale ranging from 1 (better than usual) to 4 (much worse than usual). Scores are summed, with higher scores indicating higher severity of fatigue. The BFS demonstrates high internal reliability and reasonable discriminant validity. We used the revised 11-item version, which was shown to have a Cronbach's alpha of 0.89 [36], high sensitivity (92\%) and negative predictive value (94\%) toward detecting severely high fatigue among cancer patients (i.e., scores of 11 or above; [37]). The reliability of the BFS for the current sample ranged from 0.85 to 0.92 .

\section{Concerns About Recurrence Scale}

Breast cancer survivors completed Concerns About Recurrence Scale (CARS) [38], which measures one's fear of disease recurrence. CARS is a 30-item inventory divided into five types of worries related to a potential cancer recurrence: Death worries, Health worries, Role worries, Womanhood worries and Overall Fear of Recurrence. With the exception of the four items assessing Overall Fear of Recurrence (on a scale of 1 to 6), participants rated the remaining 26 items on a scale of 1 (not at all) to 4 (extremely). Higher scores indicate more concerns and fears. The four subscales and overall fear index are reported to have high internal consistency $(\alpha=0.87$ to 0.94$)$. For the present study, CARS was shown to be highly reliable $(\alpha=0.95$ for Chinese breast cancer survivors and 0.96 for White breast cancer survivors).

\section{Perceived Stress Scale}

The Perceived Stress Scale (PSS) [39] is a 14-item questionnaire that measures stress-related frequency occurrence over the past month (e.g., 'in the last month, how often have you been upset because of something that happened unexpectedly?'). Participants rated each item on a scale ranging from 0 (never) to 4 (very often). Scores are summed, with higher scores indicating a higher degree of stress experienced during the past month. The reliability of the PSS ranges between $\alpha=0.84$ to 0.86 . For the current sample, Cronbach's alpha ranged from 0.77 to 0.84 , depending on group.

\section{Daily Stress Inventory}

The Daily Stress Inventory (DSI) [40] is a 58-item inventory that describes events that participants may have experienced within the past $24 \mathrm{~h}$. If relevant, they are asked to rate the degree of stress they experienced on a scale 
ranging from 1 (Occurred but was not stressful) to 7 (caused to panic). The Average Impact Rating scores (AIR; sum of total impact scores divided by number of events) were used for our analyses. Brantley et al. [40] reported that the DSI has a Cronbach's alpha above 0.80 , and AIR scores demonstrated adequate concurrent validity with a global rating of stress $(\mathrm{r}=0.49 ; \mathrm{p}<0.01)$. For the current sample, the DSI had a Cronbach's alpha ranging from 0.85 to 0.98 .

\section{Visual Analog Scale}

The Visual Analog Scale (VAS) is a $100 \mathrm{~mm}$ bipolar line that can be used to measure the intensity of any given characteristic on a continuum ranging from 0 to 100. Participants indicated their level of subjective feelings of stress [41] through the statement 'I feel stressed'. Scores were determined by measuring the distance from 0 (not at all) to the appraisal mark, up to 100 (very much).

\section{Trier social stress test}

The Trier Social Stress Test (TSST) is a commonly used procedure to induce moderate psychological stress in a laboratory setting [27]. The TSST consists of two tasks: a 5 min mock job interview in front of a panel of three confederates, which in our case included at least one male and one female and at least one White and one Chinese individual, and a 5 min arithmetic task that required participants to count backward sequentially by 13 from 1022. Throughout the TSST, confederates were instructed to remain neutral and to provide no verbal or nonverbal feedback to the participant.

\section{Salivary cortisol}

To measure diurnal and acute cortisol reactivity, a total of 17 saliva samples were collected from participants. Participants were instructed to collect ten samples at home over two consecutive days to serve as a diurnal assessment. Seven additional saliva samples were collected during the laboratory session to measure acute cortisol reactivity. Saliva samples were aliquoted into Eppendorf tubes and stored in a freezer at $-80^{\circ} \mathrm{C}$ until processed. Saliva samples were assayed for cortisol using enzyme-linked immunosorbent assay (ELISA) kits following a protocol developed by Salimetrics, State College, PA [42]. Samples were assayed in duplicate and the average cortisol concentration was used for the analyses.

\section{Procedure}

\section{Preliminary consultation \& diurnal saliva collection}

Eligible participants were invited to a preliminary consultation to provide more information on the study and instructions for at-home (diurnal) saliva collection. Participants received a kit, which included an insulated lunch bag, an ice pack, ten prelabeled salivettes to use at each timepoint (Waking, 30 min after waking, 12 PM, 4 PM and $9 \mathrm{PM}$ each of the 2 days), reminder instructions and a compliance recording booklet.

In preparation for the diurnal collection, participants were instructed to not ingest any alcohol $24 \mathrm{~h}$ prior to sample collection. They were also asked to refrain from exercising, brushing their teeth, ingesting caffeinated products and/or having a large meal $1 \mathrm{~h}$ prior to sample collection. The collection procedure involved participants rinsing their mouths with water $10 \mathrm{~min}$ prior to sampling, placing the swab underneath their tongues for $3 \mathrm{~min}$ and after $3 \mathrm{~min}$, to expectorate the swab back into the salivette and refrigerate it immediately or keep it cool by using the provided insulated lunch bag and ice pack. Participants also completed a set of compliance questions paired with each collection timepoint. To document their perceived stress level at the time of saliva collection, participants indicated on a VAS their stress level based on the statement 'I feel stressed' after each collection.

\section{Laboratory session}

The laboratory session was typically $2 \mathrm{~h}$ in length and was scheduled within a month of the first meeting, between 3:30 and 6:30 PM. As shown in Figure 1, the laboratory session comprised two parts: the TSST and the questionnaire component. Upon arrival, each participant indicated on the VAS how stressed she felt in order to determine whether a stressor was experienced prior to the laboratory session, which may have biased later results. Participants were offered a cup of water to drink, followed by a 10-min acclimatization period. After 10 min, the first of the seven additional saliva samples were collected: Arrival, Anticipation, Arithmetic, 10-, 20-, 40- and 60-min after TSST. The participant also indicated on the VAS how stressed she felt at the time of each collection. The 'Arrival' sample served as a baseline cortisol measurement and subjective stress prior to the acute stressor. 


\section{Cross-cultural analysis of salivary cortisol}

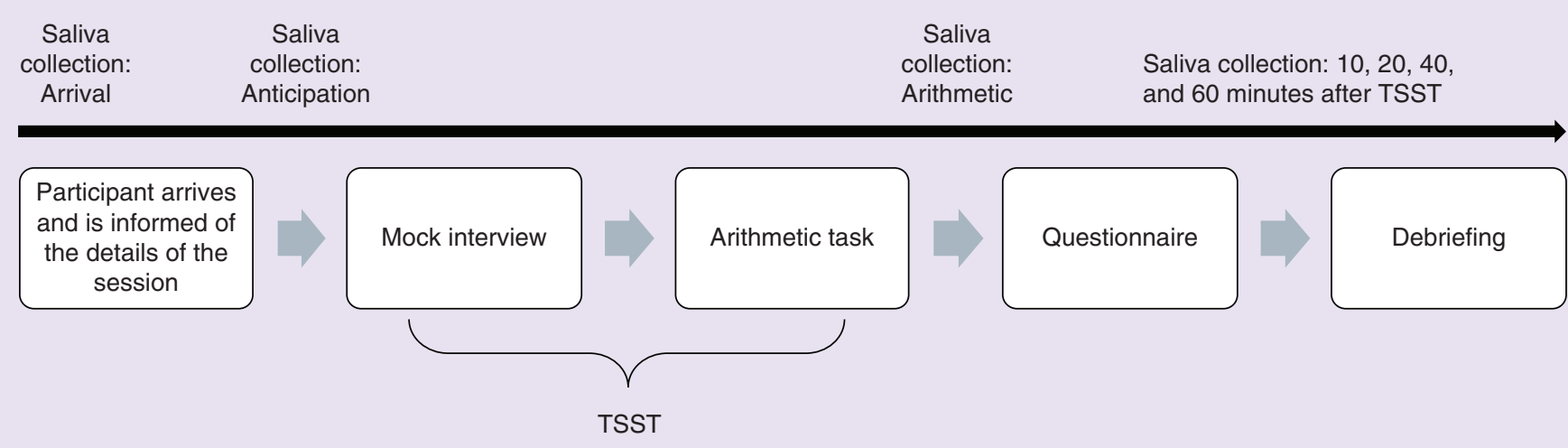

Figure 1. Schematic diagram of laboratory timeline. Laboratory sessions were approximately $1.5-2 \mathrm{~h}$ in length, and seven saliva samples were collected throughout the session. At each saliva collection, participants were required to indicate their level of subjective stress on a visual analog scale.

TSST: Trier social stress test.

After, the participant was introduced to the TSST tasks and a second saliva collection, 'Anticipation,' was collected immediately before they began the first task of the TSST, to serve as a measure of anticipatory stress. Immediately after the TSST, 'Arithmetic' was collected to assess stress patterns after the acute stressor, followed by the remaining four saliva collections at the pre-determined time points of the recovery period. The questionnaires were also completed during the recovery period (i.e., 10 to 60-min following the TSST), and participants were instructed to complete the questionnaires based on their general stress, not in relation to the TSST. After the last sample collection, the participant was debriefed of the study and compensated $\$ 50$ for her involvement in the study.

\section{Data preparation \& covariate considerations}

Missing data were imputed using EM algorithm on SPSS V23. Biomarker data were imputed for participants with up to two missing diurnal cortisol data points and up to three missing acute cortisol data points, inclusive of removed univariate outliers. Of the total number of data points collected across participants, $2.1 \%$ of diurnal cortisol values and $4.8 \%$ of acute cortisol values were imputed. Eight participants were removed due to significant missing diurnal and acute cortisol data.

Participants were excluded from analyses if they had $15 \%$ or more missing responses on any of the questionnaires, inclusive of removed univariate outliers (no bivariate outliers were identified). 12 participants were excluded from the analyses for this reason. Approximately $2 \%$ of the total questionnaire data were imputed (less than $1 \%$ for each group). The majority of the scales were normally distributed, with the exception of the responses on the Bidimensional Fatigue Scale for the White control group. We opted to not transform any of the scales because they were normally distributed for the other groups. The final sample included 27 Chinese control participants, 31 White control participants, 17 Chinese breast cancer survivors and 24 White breast cancer survivors.

Based on the literature, we selected the following eight variables to test as possible covariates for an analysis of covariance (ANCOVA): age, number of years in Canada, cultural orientation, mental and physical fatigue, time since diagnosis, stage of diagnosis, fear of recurrence and perceived stress (i.e., PSS score). None of the selected variables met the assumptions of covariates for either diurnal or acute salivary cortisol data [43]; therefore, no covariates were incorporated in the analyses.

\section{Data analysis plan}

\section{Sociodemographic, medical \& cultural orientation variables}

Following data preparation, a series of $2 \times 2$ multivariate analysis of variance (MANOVA) and chi-squares were conducted to determine whether there were group differences (ethnocultural and health statuses) in various sociodemographic and medical characteristics. Variables entered in the two-way MANOVA included 'age' and 
'number of years in Canada.' We also computed a chi-square analysis to detect significant differences in the following sociodemographic variables between groups: whether one was born in Canada, relationship status, level of education, employment status, annual family income, average of alcohol and caffeine consumed per day and smoking (yes/no).

Similar analyses were conducted to determine group differences in medical characteristics between Chinese and White breast cancer survivors. Specifically, an ANOVA was computed to assess whether there were group differences in age at diagnosis. A chi-square analysis was also used to determine whether there were group differences in stage of diagnosis, type of surgery, types of therapy and surgery (radiation, chemotherapy, hormone, breast reconstruction) and whether one had a breast cancer recurrence.

A one-way MANOVA was also computed to assess whether there were differences in medical characteristics between Chinese and White breast cancer survivors. An additional $2 \times 2$ MANOVA was performed to determine whether there were significant differences in degree of cultural orientation toward the dominant (Canadian) and non-dominant (Chinese) cultures between Chinese and White participants.

\section{Physiological stress}

The physiological data were analyzed via a mixed-design $2 \times 2 \times 5$ and $2 \times 2 \times 7$ ANOVA to assess whether there were ethnocultural group and health status differences in average cortisol concentrations across an average of five diurnal collection timepoints collected over a 2-day period (at Waking, 30 min after waking, 12 PM, 4 PM and 9 PM) and seven acute stress collection timepoints (Arrival, Anticipation, Arithmetic, 10-, 20-, 40- and 60-min). In addition, we also performed a series of $2 \times 2$ ANOVAs to assess whether there were significant differences between ethnocultural group and health status in diurnal and acute cortisol concentrations as determined by area under the curve from increase $\left(\mathrm{AUC}_{\mathrm{i}}\right)$.

\section{Psychosocial variables in relation to physiological stress}

Mixed-design $2 \times 2 \times 5$ and $2 \times 2 \times 7$ ANOVAs were performed to assess whether participants' perception of stress, as measured via the VAS, differed significantly during the diurnal and acute stress periods. Additional post-hoc two-way ANOVAs were performed to further explore significant interactions. In addition to the main VAS analyses, we also computed a $2 \times 2$ ANOVA to examine whether there were ethnocultural group or health status differences in the perception of stress (as determined by the VAS) at the onset of the acute stressor in comparison to their baseline response (i.e., Arithmetic minus Arrival).

For the other psychological stress measures, namely the perceived stress scale (PSS) and daily stress inventory (DSI), two separate 2 (ethnocultural group) $\times 2$ (health status) ANOVAs were performed. Specifically, the analyses were computed to detect group differences in total perceived stress within the past month as measured via the PSS, and the calculated average impact rating of daily stressors as determined by the DSI.

Finally, a series of $2 \times 2$ mixed-design MANOVAs were conducted to evaluate whether there were ethnocultural group or health status differences in reported levels of fatigue (physical, mental and total) and fear of recurrence (overall fears, health worries, womanhood worries, role worries and death worries).

\section{Results}

Sociodemographic \& medical characteristics

Participants' sociodemographic characteristics are displayed in Supplementary Table 1. Results revealed a significant difference among ethnocultural groups, $F(2,31)=5.78$, Pillai's Trace $=0.27, \eta_{\mathrm{p}}{ }^{2}=0.271$ and health status, $F(2$, $31)=5.56, \mathrm{p}=0.009$, Pillai's Trace $=0.26, \eta_{\mathrm{p}}{ }^{2}=0.264$, but no interaction between the sociodemographic variables. Specifically, White participants had been in Canada longer than Chinese participants $(M=36.97$ years, $\mathrm{SE}=4.53$ vs $\mathrm{M}=19.94$ years, $\mathrm{SD}=2.69 ; \mathrm{p}=0.003)$ and breast cancer survivors were significantly older than control participants $(\mathrm{M}=58.85, \mathrm{SE}=2.86$ vs $\mathrm{M}=45.33$ years, $\mathrm{SE}=2.82 ; \mathrm{p}=0.002)$. Significantly more White participants were born in Canada than Chinese participants $(\mathrm{p}<0.001)$ and the majority of Chinese and White participants reported being in a civil union or married relationship $(\mathrm{p}=0.014)$. White participants also reported higher caffeinated consumption per day than Chinese participants $(\mathrm{p}<0.001)$. On average, participants typically reported white collar occupations, with a higher proportion of control participants in white collar occupations than breast cancer survivors (65 vs 35\%; $\mathrm{p}=0.002$ ). As shown in Supplementary Table 2, Chinese and White breast cancer survivors exhibited similar medical characteristics with no significant differences found. 


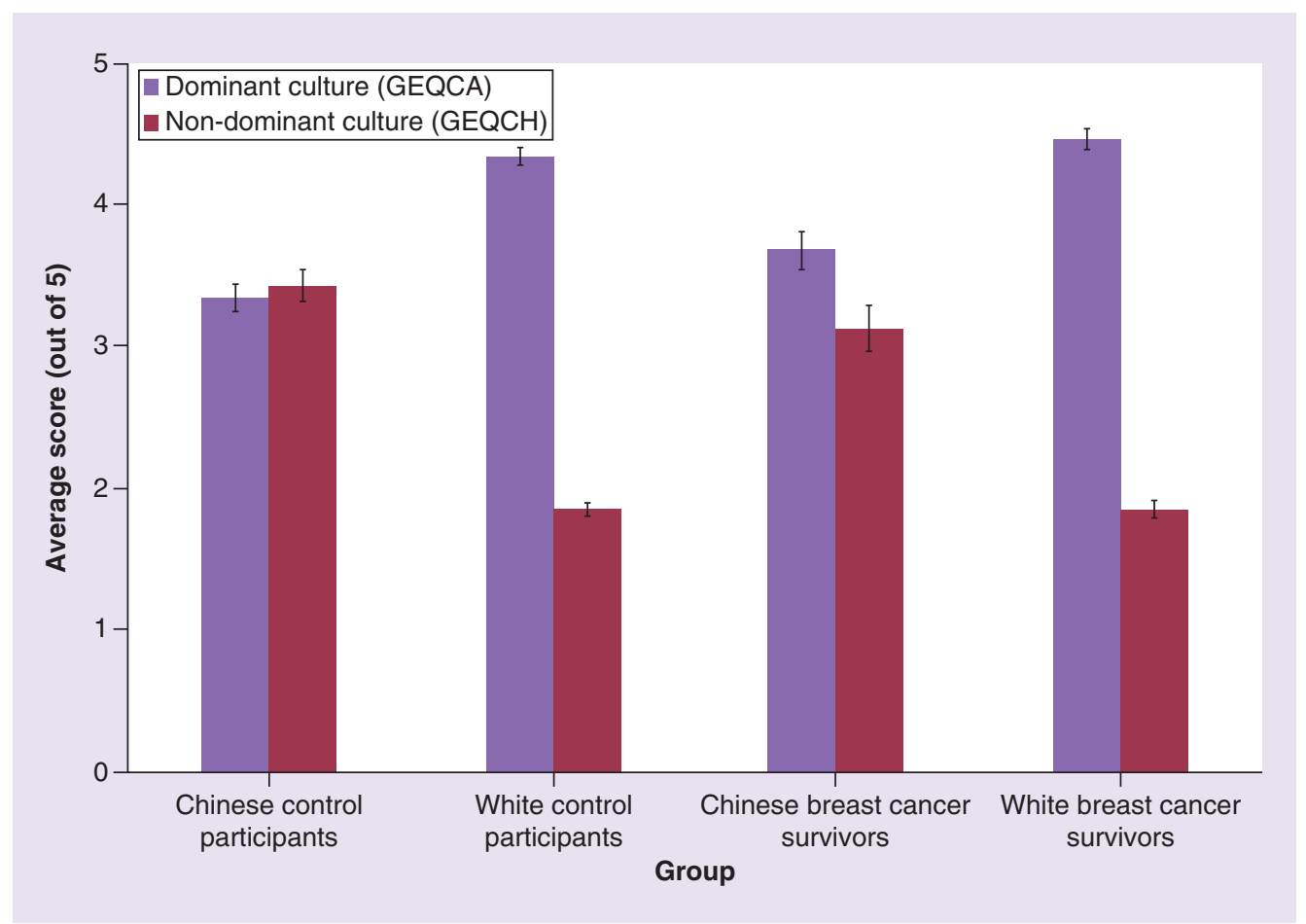

Figure 2. Degree of orientation toward dominant (Canadian, GEQCA) and non-dominant (Chinese, GEQCH) cultures. Error bars represent standard error of the mean.

\section{Degree of cultural orientation}

Figure 2 shows participants' degree of cultural orientation toward the dominant (Canadian) and non-dominant (Chinese) cultures. Significant differences were found between ethnocultural groups, F(2, 94) $=113.38 ; p<0.001$, Pillai's Trace $=0.71, \eta_{\mathrm{p}}{ }^{2}=0.71$ and health status, $\mathrm{F}(2,94)=3.22 ; \mathrm{p}=0.044$, Pillai's Trace $=0.06, \eta_{\mathrm{p}}{ }^{2}=0.06$, but no significant interaction. Although participants were typically more oriented toward their own respective culture, results revealed that breast cancer survivors (irrespective of ethnocultural group) were significantly more oriented toward the dominant culture than was observed in control participants $(\mathrm{M}=3.84, \mathrm{SE}=0.06$ vs $\mathrm{M}=4.06, \mathrm{SE}=$ $0.07)$.

\section{Compliance}

Participants were deemed non-compliant if they ingested alcohol within $24 \mathrm{~h}$ of saliva collection, or if they ingested a large meal, exercised or brushed their teeth within $1 \mathrm{~h}$ of saliva collection. Furthermore, participants were marked as non-compliant if samples were not collected within $30 \mathrm{~min}$ of the collection time. The majority of participants complied for the earlier sample collection times (88.9-92.9\%), but their compliance declined for the 9 PM collections (Day 1: 80.8\%, Day 2: 78.8\%).

Specifically, on both days, participants generally collected the Waking samples between 6:39 AM and 7:08 AM (median time: 6:30 AM to 7:15 AM; SD: 49 to $86 \mathrm{~min}$ ) and they were typically on time for the second collection (30 min after waking; average time: 7:14 AM to 7:44 AM; median time: 7:01 AM to 7:45 AM; SD: 49 to 72 min). Compliance reports also indicated that participants typically collected the $12 \mathrm{PM}$ and 4 PM collections on time (average times: 12:02 PM to 12:10 PM, and 4:01 PM to 4:14 PM; median time: 12:00 PM to 12:07 PM, and 4:00 PM to 4:10 PM; SD: 7 to $23 \mathrm{~min}$, and 5 to $30 \mathrm{~min}$, respectively). Compliance for 9 PM collections declined, however, and on average, they were collected between 8:32 PM and 9:23 PM (median time: 9:00 PM to 9:05 PM, SD: 14 to $247 \mathrm{~min})$. 

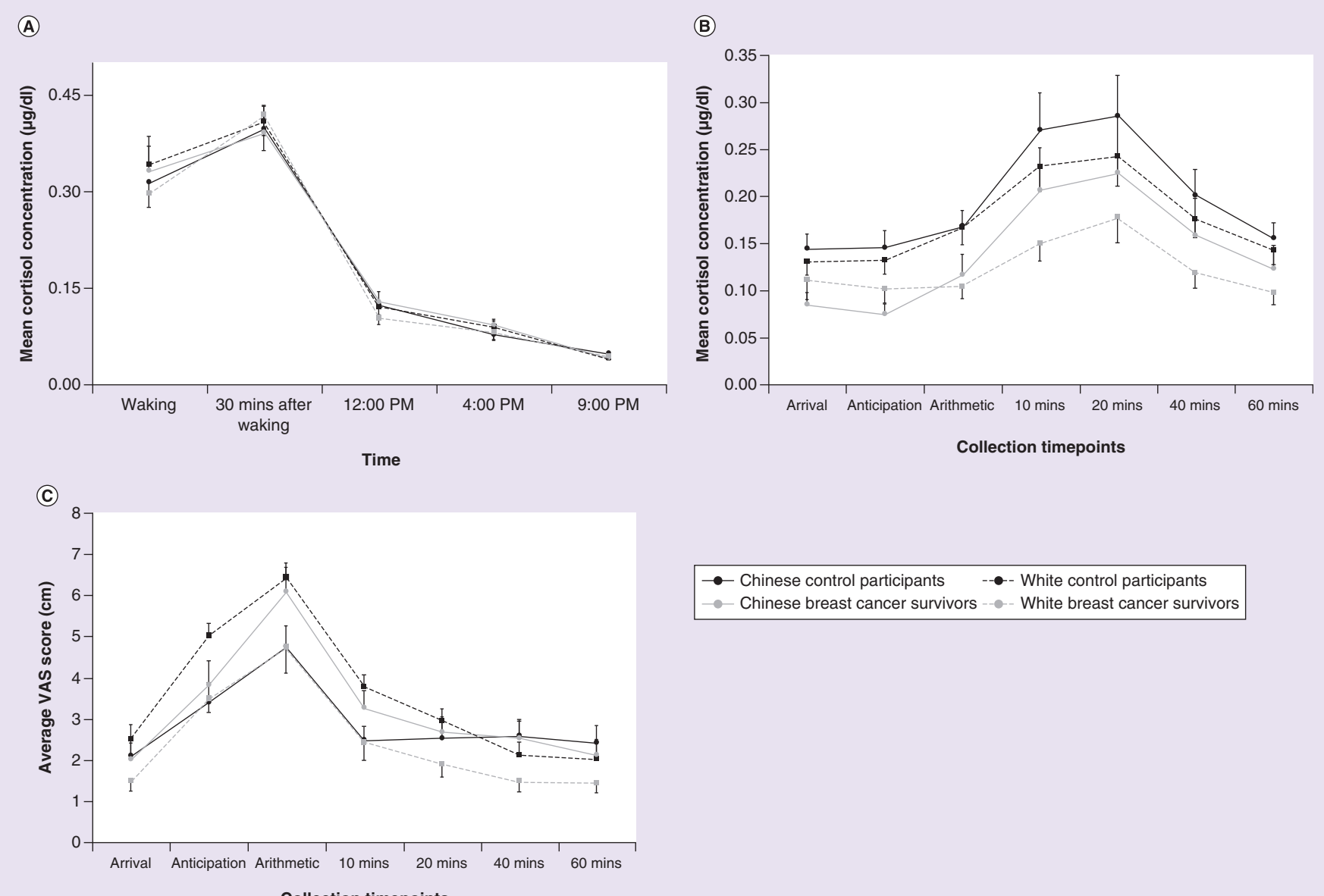

- Chinese control participants
- Chinese breast cancer survivors - White control participants
White breast cancer survivors

Collection timepoints

Figure 3. Participants' overall physiological and psychological stress profiles. (A) Mean diurnal cortisol concentration. (B) Mean acute cortisol concentration. (C) Participants' average score on the statement 'I feel stressed' as indicated on the Visual Analog Scale (0-10 cm) at each saliva collection time point of the laboratory session. Error bars represent standard error of the mean.

\section{Physiological stress profiles \\ Diurnal cortisol patterns}

Average cortisol concentrations of Day 1 and Day 2 can be found in Supplementary Table 3 (raw data presented); collapsed mean cortisol concentrations across both days are graphed in Figure 3A (cleaned data as used in analyses). As shown in Figure 3A, regardless of ethnocultural membership, breast cancer survivors and control participants displayed similar diurnal cortisol patterns. Results revealed no group differences or interactions between ethnocultural group and health status, time and ethnocultural membership or time and health status ( $\mathrm{ps}=0.509-0.994)$. The three-way interaction was not significant $(\mathrm{p}=0.547)$. Note that there were also no significant group differences in the cortisol secretion at awakening (usually referred to as the cortisol awakening response or CAR) based on the first two data time points ( $\mathrm{ps}=0.267$ to 0.663 ).

\section{Acute cortisol patterns}

As depicted in Supplementary Table 3 and Figure 3B, a significant quadratic trend was revealed in the acute cortisol patterns, $\mathrm{F}(1,90)=47.79 ; \mathrm{p}<0.001, \eta_{\mathrm{p}}{ }^{2}=0.35$. Although the main effect of ethnocultural membership was not significant $(\mathrm{p}=0.344)$, there was a significant main effect of health status, $F=6.50, p=0.012, \eta_{p}{ }^{2}=0.067$. Specifically, breast cancer survivors displayed lower cortisol concentration values than that of control participants. There were no significant two- or three-way interactions between time, ethnocultural group or health status (ps ranged from 0.161 to 0.951 ). 
Although Chinese breast cancer survivors displayed a lower Arrival cortisol concentration level than that of White breast cancer survivors, the slope of increase in cortisol reactivity of Chinese breast cancer survivors was greater than that of White breast cancer survivors, resulting in a higher peak at 20-min post-TSST for Chinese breast cancer survivors. This pattern suggests that Chinese breast cancer survivors exhibited similar cortisol reactivity to that of Chinese health control participants.

Area under the curve

Results for area under the curve (AUCi) analyses of both diurnal and acute cortisol patterns revealed no significant group main effects or interactions ( $\mathrm{ps}=0.331$ to 0.867 and 0.166 to 0.407 , respectively).

\section{Psychosocial variables in relation to physiological stress}

Visual Analog Scale

Participants' typical subjective perceptions of stress during the acute stressor (i.e., TSST; Time VAS) are shown in Figure 3C; breakdown of reports (by Day) are shown in Supplementary Table 3. Results revealed a significant main effect of time VAS, $\mathrm{F}(3.61,310.52)=77.21 ; \mathrm{p}<0.001, \eta_{\mathrm{p}}{ }^{2}=0.47$ but not of ethnocultural group $(\mathrm{p}=0.868)$ or health status $(\mathrm{p}=0.203)$. Results also indicated a significant two-way interaction between ethnocultural group and health status, $\mathrm{F}(1,86)=8.35 ; \mathrm{p}=0.005, \eta_{\mathrm{p}}{ }^{2}=0.09$, and a significant three-way interaction between ethnocultural group, health status and time VAS, $\mathrm{F}(3.61,310.52)=2.77 ; \mathrm{p}=0.032, \eta_{\mathrm{p}}{ }^{2}=0.03$.

To further examine the significant three-way interaction, we assessed whether there was an interaction between health status and Time VAS on both levels of ethnocultural group and whether there was an interaction between ethnocultural group and Time VAS on both levels of health status. Results of the first follow-up analysis indicated that there was a main effect of Time VAS on both levels of ethnocultural group (ps $<0.001$ ) but no significant interactions ( $\mathrm{ps}=0.199$ to 0.250 ). However, in a test of between-subject effects White control participants reported significantly higher average levels of perceived stress on the VAS than White breast cancer survivors $(\mathrm{p}<0.001)$. No such differences were found among Chinese control participants and breast cancer survivors.

The second follow-up analysis revealed a significant main effect of Time VAS on both levels of health status, and an interaction between ethnocultural group and Time VAS in control participants, $\mathrm{F}(3.74,198)=5.55 ; \mathrm{p}<0.001$, $\eta_{\mathrm{p}}{ }^{2}=0.095$. No such interactions were observed among breast cancer survivors $(\mathrm{p}=0.624)$. Results suggest that White control participants reported significantly higher average levels of perceived stress than did Chinese control participants, $\mathrm{F}(1,53)=4.08, \mathrm{p}=0.049, \eta_{\mathrm{p}}{ }^{2}=, 071$, whereas White breast cancer survivors reported significantly lower levels of perceived stress than Chinese breast cancer survivors, $F(1,33)=5.27, p=0.028, \eta_{p}{ }^{2}=0.138$. Simple main effects analyses revealed that Chinese control participants reported significantly lower levels of perceived stress than White control participants at timepoints 2, 3 and 4 (i.e., Anticipation, Arithmetic and 10 min after TSST cessation; $\mathrm{ps}=0.001$ to 0.01 ).

Taken together, the results revealed no significant difference in levels of perceived stress as reported by Chinese breast cancer survivors and Chinese control participants. Further, while White control participants reported markedly higher levels of stress than White breast cancer survivors at all timepoints, there was only a difference in reported levels between White and Chinese control participants for the duration of the acute stressor.

We also examined whether there were ethnocultural group or health status differences in the perception of stress at the onset of the acute stressor in comparison to their baseline response (i.e., VAS scores at Arithmetic minus Arrival). Results did not reveal significant main effects but a significant interaction between the two factors was found, $F(1,89)=3.97, p=0.049, \eta_{\mathrm{p}}{ }^{2}=0.043$. Simple main effects analyses indicated that there were no significant differences in ethnocultural group on either level of health status, and vice versa ( $\mathrm{ps}=0.053$ to 0.344 ). These results were corroborated by follow-up independent t-tests (White control participants vs White breast cancer survivors, $\mathrm{p}=0.376$; Chinese control participants vs Chinese breast cancer survivors; $\mathrm{p}=0.053$ ). Therefore, despite the interaction, there appears to be no significant statistical difference between subjective levels of stress at the onset of the acute stressor and baseline.

\section{Other psychological measures of stress}

Results revealed a significant main effect of ethnocultural group, $\mathrm{F}(1,95)=4.14, \mathrm{p}=0.045, \eta_{\mathrm{p}}{ }^{2}=0.04$. Specifically, Chinese participants, regardless of health status, reported experiencing significantly more perceived stress than did White participants $(M s=25.04$ vs 22.26). There was neither a significant main effect of health status $(\mathrm{p}=0.810)$ nor a significant interaction between the two factors $(\mathrm{p}=0.112)$. 


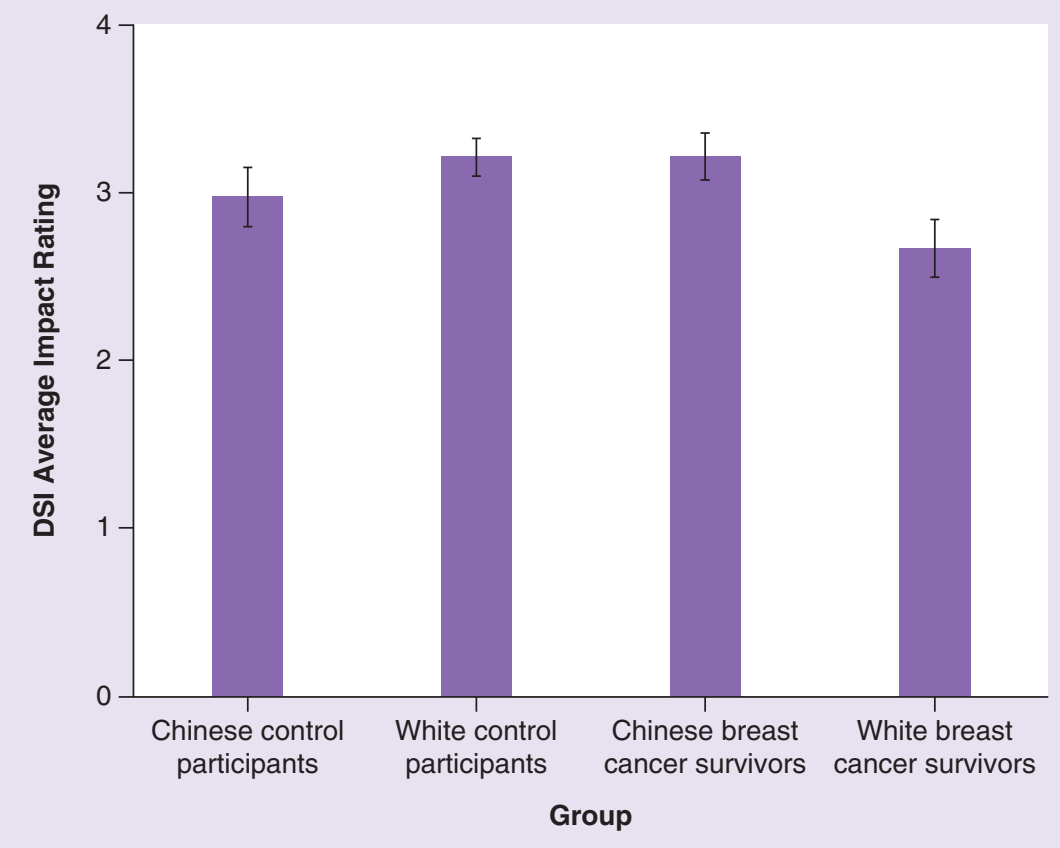

Figure 4. Participants' mean Average Impact Rating on the Daily Stress Inventory. Error bars represent standard error of the mean. DSI: Daily Stress Inventory.

Results of participants' AIR of daily stress (as determined by the DSI) revealed no significant main effects of ethnocultural group or health status ( $\mathrm{ps}=0.326$ and 0.339 , respectively), as depicted in Figure 4, there was a significant interaction between the two factors, $F(1,95)=6.06 ; p=0.016, \eta_{p}{ }^{2}=0.06$. While three of the four groups reported similar AIR, markedly lower AIR values were $(M=2.67)$ disclosed by White breast cancer survivors.

\section{Fatigue \& concerns about cancer recurrence}

Analyses did not reveal any significant group differences or interactions between ethnocultural groups and health status for physical, mental or total fatigue (ps ranged from 0.079 to 0.511 ) or for fear of recurrence (ps ranged from 0.069 to 0.988 ).

\section{Discussion}

The present study investigated whether Chinese breast cancer survivors exhibit different diurnal and acute cortisol patterns in comparison to that of healthy Chinese women and White women with and without a prior diagnosis of breast cancer. In line with previous findings, diurnal profiles did not differ between groups [24,44]. But atypical acute cortisol patterns were found, suggesting changes in the HPA stress mechanisms of breast cancer survivors, possibly due to the chronic activation of the HPA axis throughout the disease trajectory [23,24].

All groups indicated similar patterns of subjective stress appraisal during the TSST protocol (i.e., VAS stress ratings). They showed increasingly higher levels of psychological stress during the Anticipation and Arithmetic phases of the protocol and a gradual decrease in psychological stress levels during the recovery period (10 to $60 \mathrm{~min}$ after) with some minor differences across the groups (see Figure 3C). Although our VAS results were inconsistent with previous findings [24], the differences in psychological profiles of stress observed in our study may be explained by cultural factors or posttraumatic growth.

The added burden of acculturative stress may have enhanced the acute stress reactivity of Chinese participants, such that Chinese participants exhibited augmented acute stress responses irrespective of health status. Indeed, our findings indicated that Chinese women may respond to acute stress differently than that of White women. In the event of an acute stressor, while the cortisol reactivity slopes among Chinese breast cancer survivors and Chinese control participants were similar, their reactivity slopes were steeper than that of their western counterparts. Thus, 
indicating that there may be an association between acculturation, ethnocultural membership status and cortisol reactivity slopes, but this relationship needs to be further explored.

An individual's understanding and perception of the illness is central to improving her well-being and subjective feelings of stress; to do this, the role of culture-bound values, beliefs and perceptions of illness need to be considered [45]. Cultural values often influence an individual's understanding of their illness and disease trajectory [45,46]. Our study indicated that there was a significant interaction between ethnocultural membership, health status and perceived stress. However, despite the differences in the perception of stress, upon closer investigation, it was revealed that Chinese women with and without a prior diagnosis of breast cancer exhibited similar patterns of acute cortisol reactivity, while White control participants exhibited higher levels of cortisol at all timepoints than that of White breast cancer survivors and only higher levels than that of Chinese control participants during the acute stressor. Thus, our results may be indicative of posttraumatic growth, particularly among White breast cancer survivors.

It has often been reported that survivors of trauma, including medical illnesses such as breast cancer, experience posttraumatic growth, a positive psychological change due to an adverse experience [47]. The relationship between post-traumatic growth, stress appraisal and overall well-being is convoluted however. In addition to post-traumatic growth, many studies have noted that survivors of trauma also often report a re-evaluation of their core beliefs and values, the adoption and use of various coping mechanisms and the availability and use of social support - all factors that contribute toward improving their well-being and promoting resilience toward future challenges [47,48]. Supporting this literature, many breast cancer survivors in the present study anecdotally expressed changes in their perception and appraisal of stressors after their breast cancer diagnosis and treatment, such as accepting the illness as part of their new identity, adoption of new beliefs and reappraisal of their values.

The similarity in reactive cortisol profiles among Chinese breast cancer survivors and control participants may be indicative of the influences of differences in cultural values and culture-related stress. Studies have suggested that values associated with eastern or collectivistic cultures, such as greater social constraints toward discussing one's illness, hesitation toward seeking social support and fatalism, negatively affect survivorship outcomes and promote stress-related symptoms [46]. In addition to cultural differences in illness management and perception, Chinese women also endure various acculturative stressors, regardless of their degree of acculturation. Although Chinese control participants in the present study typically demonstrated bicultural orientation or, in the case of Chinese breast cancer survivors, a higher degree of orientation toward the Canadian culture, they may still experience various acculturative stressors, such as the reconciliation of different cultural beliefs and values, discrimination or rejection from in-groups and out-groups and intergenerational conflicts [30,31]. Acculturative stress, in addition to health-related stress, may therefore contribute to the observed higher subjective stress appraisals among Chinese breast cancer survivors than that indicated by White breast cancer survivors. This notion is supported by the results of two other subjective measures of stress used in this study - the Perceived Stress Scale and the Daily Stress Inventory - both of which showed that Chinese participants, regardless of health status, typically reported feeling upset, stressed, angry or nervous more often than did White participants within the past month.

\section{Limitations}

Although our results replicated previous findings regarding physiological stress responses toward an acute stressor, unlike earlier studies [24], our findings also suggested a blunting effect in psychological patterns of stress. We offered potential interpretations of the discrepant findings on psychological and physiological stress profiles; however, further research is required as the findings may be spurious or due to cohort effects. In particular, the breast cancer survivors in the present study were on average 10-12 years older than their healthy counterparts and, as noted by the literature, age may affect one's HPA functioning and stress appraisal. However, our results are consistent with other similar studies [21-26], which included breast cancer survivors of varying ages; thus, it is unlikely that observed differences in cortisol response were due to the age difference. Another potential cohort effect is geographical location; our participants were recruited from large Canadian urban centers (Ottawa and Toronto) where different cultures are shared and respected. Therefore, Chinese participants in the present study may have different experiences than that of Chinese women in rural Canadian towns or cities in China, where individuals may not be immersed in a multicultural environment.

We also acknowledge that there are several other limitations to our study, such as its cross-sectional design, small sample size and stringent eligibility criteria; therefore, our results should be interpreted with caution. One important methodological consideration may be the order in which questionnaires are presented in relation to 
the acute stressor. In the present study, questionnaires were administered after the TSST, which may potentially introduce bias in subjective measurements of stress even though participants were explicitly instructed to not account for the TSST experience during the completion of the questionnaires. However, the administration of questionnaires prior to the acute stressor may also introduce other limitations, such as the priming of participants to be more emotionally agitated or distressed, thus biasing the results of the TSST. Hence, it is recommended that future studies explore and control for possible order effects of the administration of subjective measures of stress on cortisol release.

In addition, acculturative effects may also have been obscured due to the inclusion requirement of English comprehension, which may have prevented our study from capturing the full spectrum of acculturative stress. Participants in the present study were also mostly older adults who were well-educated, married and employed with mid- to high-income, thus precluding them from stressors that may be associated with members of other socioeconomic and demographic characteristics (e.g., inability to pay bills, child rearing, lack of job security). Therefore, our findings may not be generalizable and representative of the population, especially within the Chinese community.

Future studies pursuing this avenue of research should recruit a larger sample that is more diverse in their sociodemographic characteristics and degree of acculturation (i.e., number of years in Canada and at various points of the acculturative process). It would also be useful to include more measures to examine other aspects of acculturation, such as perceived discrimination, family functioning and perception of illness, as well as appraisal of stressors as it relates to one's culture. Indeed, discrimination and family conflict have often been reported to negatively affect the psychological and physiological well-being of second generation adolescents $[49,50]$.

Considering the extent of multiculturalism and ethnic diversity within Canada, it is important to increase health providers' awareness of the implications of cultural beliefs and values, which will likely improve and promote a positive survivorship experience for patients of varying ethnicities. Recent developments in symptom management services and programs allow survivors to access support services more readily; however, the existing programs and services are still heavily influenced by Western beliefs. Given the influence of cultural beliefs and values on the perception of illness, appraisal of stressors and survivorship outcomes [46], future studies are encouraged to further investigate and develop culturally appropriate treatment plans and post-treatment services.

\section{Conclusion}

To our knowledge, this is the first study to provide a physiological and psychological account of stress between Chinese and White women with and without a prior diagnosis of breast cancer. Our results replicated previous findings in physiological patterns of stress; regardless of ethnocultural membership, when compared with control participants, breast cancer survivors exhibited a blunted acute cortisol response despite no differences in diurnal cortisol profiles. But contrary to previous studies, we also found group differences in psychological profiles of stress. Although further investigation is warranted, our study provides preliminary evidence that ethnocultural membership and cultural orientation may influence individuals' appraisal and perception of stress, thus affecting their immediate physiological (i.e., slope of cortisol reactivity) and psychological responses toward a stressor. Our study therefore highlights the impact of acculturative stress on physiological and psychological stress profiles and underscores the need for more research and greater understanding of the effects of culture on well-being and health.

\section{Summary points}

- Breast cancer survivors, irrespective of ethnocultural membership, exhibited a blunted cortisol response toward an acute stressor.

- A significant interaction was observed between ethnocultural group, health status and time.

- White control participants reported higher levels of subjective stress than that of Chinese control participants.

- Results suggest that cultural orientation and ethnocultural membership may affect one's perception of stress, thus highlighting the importance of cultural sensitivity and awareness among healthcare providers and existing programs. 


\section{Acknowledgments}

We would like to thank our research participants for their dedication and ongoing support.

Financial \& competing interests disclosure

The authors have no relevant affiliations or financial involvement with any organization or entity with a financial interest in or financial conflict with the subject matter or materials discussed in the manuscript. This includes employment, consultancies, honoraria, stock ownership or options, expert testimony, grants or patents received or pending, or royalties.

No writing assistance was utilized in the production of this manuscript.

\section{Ethical conduct of research}

All procedures performed in studies involving human participants were in accordance with the ethical standards of the institutional and/or national research committee and with the 1964 Helsinki declaration and its later amendments or comparable ethical standards. Informed consent was obtained from all individual participants included in the study.

Data availability

Datasets generated during and/or analysed during the current study are not publicly available, but may be obtained from the corresponding author on reasonable request.

\section{Open access}

This work is licensed under the Attribution-NonCommercial-NoDerivatives 4.0 Unported License. To view a copy of this license, visit http://creativecommons.org/licenses/by-nc-nd/4.0/

\section{References}

Papers of special note have been highlighted as: $\bullet$ of interest; $\bullet \bullet$ of considerable interest

1. Deapen D, Liu L, Perkins C, Bernstein L, Ross RK. Rapidly rising breast cancer incidence rates among Asian-American women. Int. J. Cancer 99(5), 747-750 (2002).

2. Minami Y, Tsubono Y, Nishino Y, Ohuchi N, Shibuya D, Hisamichi S. The increase of female breast cancer incidence in Japan: emergence of birth cohort effect. Int. J. Cancer. 108(6), 901-906 (2004).

3. Chia K-S, Reilly M, Tan C-S et al. Profound changes in breast cancer incidence may reflect changes into a Westernized lifestyle: a comparative population-based study in Singapore and Sweden. Int. J. Cancer 113(2), 302-306 (2005).

4. Silva ND, Dillon FR, Verdejo TR, Sanchez M, De La Rosa M. Acculturative stress, psychological distress, and religious coping among latina young adult immigrants. Couns. Psychol. 45(2), 213-236 (2017).

5. Wan C, Arès I, Gareau A, Collins KA, Lebel S, Bielajew C. Motherhood and well-being in young breast cancer survivors. Breast Cancer Manag. (2018). www.futuremedicine.com/doi/full/10.2217/bmt-2017-0015.

6. Wan C, Couture-Lalande M-È, Lebel S, Bielajew C. The role of stressful life events on the cortisol reactivity patterns of breast cancer survivors. Psychol. Health. 32(12), 1485-1501 (2017).

7. Wan C, Couture-Lalande M-Ė, Narain TA, Lebel S, Bielajew C. Salivary alpha-amylase reactivity in breast cancer survivors. Int. J. Environ. Res. Public. Health. 13, 353-367 (2016).

- The stress system comprises the hypothalamic-pituitary-adrenal axis (HPA) as well as the sympathetic-adrenal-medullary (SAM) axis, and their functioning can be measured via cortisol and alpha-amylase, respectively. To fully understand the mechanisms underlying stress, it is important to examine the synergistic relationship between the two axes. Wan et al's (2016) study complements Couture-Lalande et al.'s (2014) study in that the former examines the performance of the SAM axis whereas the latter concentrates on the HPA axis, in the same sample of participants. Therefore, this study will help readers to have a better understanding of the overall functioning of the stress system in breast cancer survivors.

8. Wan C, Silverstein K, Arès I, Bielajew C. Predictors of fear of recurrence in breast cancer survivors. Cancer Stud. Ther. 1, 1-8 (2016).

9. Wen K-Y, Fang CY, Ma GX. Breast cancer experience and survivorship among Asian Americans: a systematic review. J. Cancer Surviv. 8(1), 94-107 (2014).

10. Lazarus RS, Folkman S. Transactional theory and research on emotions and coping. Eur. J. Personal. 1(3), 141-169 (1987).

11. Nesse RM, Bhatnagar S, Ellis B. Evolutionary Origins and Functions of the Stress Response System. In: Stress: Concepts, Cognition, Emotion, and Behavior. Fink G. (Ed.), Academic Press, CA, USA, 95-101 (2016) www.sciencedirect.com/science/article/pii/B978012800951200011X.

12. Thiel KJ, Dretsch MN. The basics of the stress response: A historical context and introduction. Conrad CD. (Ed.), Wiley-Blackwell, Oxford, UK, 1-28 (2011).

13. Fulford AJ, Harbuz MS. An introduction to the HPA axis. Tech. Behav. Neural Sci. 15, 43-65 (2005). 
14. Hellhammer DH, Wüst S, Kudielka BM. Salivary cortisol as a biomarker in stress research. Psychoneuroendocrinology 34(2), 163-171 (2009).

15. Foley P, Kirschbaum C. Human hypothalamus-pituitary-adrenal axis responses to acute psychosocial stress in laboratory settings. Neurosci. Biobehav. Rev. 35(1), 91-96 (2010).

16. Stone AA, Schwartz JE, Smyth J et al. Individual differences in the diurnal cycle of salivary free cortisol: a replication of flattened cycles for some individuals. Psychoneuroendocrinology 26(3), 295-306 (2001).

17. Huang Y, Zhou R, Wu M, Wang Q, Zhao Y. Premenstrual syndrome is associated with blunted cortisol reactivity to the TSST. Stress 18(2), 160-168 (2015).

18. Kudielka BM, Hellhammer DH, Wüst $S$. Why do we respond so differently? Reviewing determinants of human salivary cortisol responses to challenge. Psychoneuroendocrinology 34(1), 2-18 (2009).

19. Buckley TM, Schatzberg AF. On the interactions of the hypothalamic-pituitary-adrenal (HPA) axis and sleep: normal HPA axis activity and circadian rhythm, exemplary sleep disorders. J. Clin. Endocrinol. Metab. 90(5), 3106-3114 (2015).

20. Lundstrom S, Furst CJ. Symptoms in advanced cancer: relationship to endogenous cortisol levels. Palliat. Med. 17(6), 503-508 (2003).

21. Dedert E, Lush E, Chagpar A et al. Stress, coping, and circadian disruption among women awaiting breast cancer surgery. Ann. Behav. Med. 44(1), 10-20 (2012).

22. Ho RTH, Fong TCT, Chan CKP, Chan CLW. The associations between diurnal cortisol patterns, self-perceived social support, and sleep behavior in Chinese breast cancer patients. Psychoneuroendocrinology 38(10), 2337-2342 (2013).

23. Alexander S, Minton O, Andrews P, Stone P. A comparison of the characteristics of disease-free breast cancer survivors with or without cancer-related fatigue syndrome. Eur. J. Cancer. 45(3), 384-392 (2009).

24. Couture-Lalande M-Ë, Lebel S, Bielajew C. Analysis of the cortisol diurnal rhythmicity and cortisol reactivity in long-term breast cancer survivors. Breast Cancer Manag. 3(6), 465-476 (2014).

-. This publication informed the design of the study. One aim of the present study was to replicate the findings based on the White sample of breast cancer survivors they described.

25. Touitou Y, Bogdan A, Levi F, Benavides M, Auzeby A. Disruption of the circadian patterns of serum cortisol in breast and ovarian cancer patients: Relationships with tumour marker antigens. Br. J. Cancer 74(8), 1248-1252 (1996).

26. Touitou Y, Lévi F, Bogdan A, Benavides M, Bailleul F, Misset J-L. Rhythm alteration in patients with metastatic breast cancer and poor prognostic factors. J. Cancer Res. Clin. Oncol. 121(3), 181-188 (1995).

27. Kirschbaum C, Pirke KM, Hellhammer DH. The 'Trier Social Stress Test' - a tool for investigating psychobiological stress responses in a laboratory setting. Neuropsychobiology 28(1-2), 76-81 (1993).

28. Miller GE, Chen E, Zhou ES. If it goes up, must it come down? Chronic stress and the hypothalamic-pituitary-adrenocortical axis in humans. Psychol. Bull. 133(1), 25 (2007).

-. Study offers a comprehensive meta-analytic overview of the HPA axis and the effects of stress on its functioning.

29. Berry JW. Acculturative Stress. Wong PTP, Wong LCJ (Eds), Springer, NY, USA, 287-298 (2006).

30. Phinney JS, Ong A, Madden T. Cultural values and intergenerational value discrepancies in immigrant and non-immigrant families. Child Dev. 71(2), 528-539 (2000).

31. Stroink ML, Lalonde RN. Bicultural identity conflict in second-generation Asian Canadians. J. Soc. Psychol. 149(1), 44-65 (2009).

32. Choi G. Acculturative stress, social support, and depression in Korean American families. J. Fam. Soc. Work. 2(1), 81-97 (1997).

33. Fang CY, Ross EA, Pathak HB, Godwin AK, Tseng M. Acculturative stress and inflammation among Chinese immigrant women. Psychosom. Med. 76(5), 320-326 (2014).

34. Logan JG, Barksdale DJ, Carlson J, Carlson BW, Rowsey PJ. Psychological stress and arterial stiffness in Korean Americans. J. Psychosom. Res. 73(1), 53-58 (2012).

35. Tsai JL, Ying Y-W, Lee PA. The meaning of "being Chinese" and "being American": variation among Chinese American young adults. J. Cross Cult. Psychol. 31(3), 302-332 (2000).

- The main cross-cultural measure used in the study was that of cultural orientation, which was developed by Tsai et al. (2000). This article provides more information regarding the measure as well as their preliminary findings during the validation of the measure.

36. Chalder T, Berelowitz G, Pawlikowska T et al. Development of a fatigue scale. J. Psychosom. Res. 37(2), 147-153 (1993).

37. Alexander S, Minton O, Stone PC. Evaluation of screening instruments for cancer-related fatigue syndrome in breast cancer survivors. J. Clin. Oncol. 27, 1197-1201 (2009).

38. Vickberg SM. The Concerns About Recurrence Scale (CARS): Asystematic measure of women's fears about the possibility of breast cancerrecurrence. Ann. Behav. Med.25(1), 16-24 (2003).

39. Cohen S, Kamarck T, Mermelstein R. A global measure of perceived stress. J. Health Soc. Behav. 24(4), 385-396 (1983). 
40. Brantley PJ, Waggoner CD, Jones GN, Rappaport NB. A daily stress inventory: development, reliability, and validity. J. Behav. Med. 10(1), 61-74 (1987).

41. Aitken RC. Measurement of feelings using visual analogue scales. Proc. R. Soc. Med. 62(10), 989-993 (1969).

42. Salimetrics. 2014 Expanded range high sensitivity salivary cortisol enzyme immunoassay kit.

43. Tabachnick BG, Fidell LS. Using Multivariate Statistics (6e). Pearson Education, NJ, USA. (2013)

44. Hsiao F-H, Jow G-M, Kuo W-H et al. The effects of psychotherapy on psychological well-being and diurnal cortisol patterns in breast cancer survivors. Psychother. Psychosom. 81(3), 173-182 (2012).

45. Cordova MJ, Giese-Davis J, Golant M, Kronenwetter C, Chang V, Spiegel D. Breast cancer as trauma: posttraumatic stress and posttraumatic growth. J. Clin. Psychol. Med. Settings 14(4), 308-319 (2007).

46. Ashing-Giwa KT, Padilla G, Tejero J et al. Understanding the breast cancer experience of women: a qualitative study of African American, Asian American, Latina and Caucasian cancer survivors. Psycho-Oncology 13(6), 408-428 (2004).

47. Jim HSL, Jacobsen PB. Posttraumatic stress and posttraumatic growth in cancer survivorship: a review. Cancer J. 14(6), 414-419 (2008).

48. Diaz M, Aldridge-Gerry A, Spiegel D. Posttraumatic growth and diurnal cortisol slope among women with metastatic breast cancer. Psychoneuroendocrinology 44, 83-87 (2014).

49. Busse D, Yim IS, Campos B, Marshburn CK. Discrimination and the HPA axis: current evidence and future directions. J. Behav. Med. 40(4), 539-552 (2017).

50. Crane DR, Ngai SW, Larson JH, Hafen M. The influence of family functioning and parent-adolescent acculturation on North American Chinese adolescent outcomes. Fam. Relat. 54(3), 400-410 (2005). 
